\title{
Synthesis and Characterization of Novel Series of 1,2,4-Triazolo[3,4-b]-1,3,4-Thiadiazole Derivatives of Anilinoacetic Acids as Promising Antioxidant Agents
}

\author{
Jean Baptiste Nkurunziza and Balakrishna Kalluraya* \\ Department of Studies in Chemistry, Mangalore University, India
}

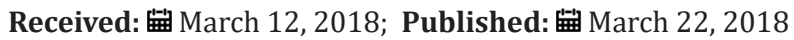

*Corresponding author: Balakrishna Kalluraya, Department of Studies in Chemistry, Mangalore University, India, Tel: 91-9448824075;

Email: bkalluraya@gmail.com

\begin{abstract}
The preparation of a novel series of 6-arylaminomethyl-3-substituted-1,2,4-triazolo[3,4-b]-1,3,4-thiadiazoles (5) by the cyclocondensation of 5-substituted-4-amino-3-mercapto-1,2,4-triazoles (2) with appropriately substituted anilinoacetic acids (4) employing phosphorous oxychloride is reported. The newly synthesized compounds (5) were screened for their antioxidant activity by DPPH radical scavenging assay and their structures were elucidated on the basis of their ${ }^{1} \mathrm{H}-\mathrm{NMR}$, IR, Mass spectral and elemental analysis. Some of the tested compounds showed good DPPH radical scavenger activities comparable to that of standard BHA.
\end{abstract}

Keywords: Anilinoacetic acids; 1,2,4-Triazoles; Triazolo-thiadiazoles; Antioxidant activity

\section{Introduction}

The 1,2,4-triazole nucleus constitutes an integral component in drug discovery. It has been incorporated into both non-bridged drugs such as anastrozole (anticancer) [1,2], ribavirin (antiviral) $[1,3]$, fluconazole, voriconazole (antifungal agents) [4] and in biologically active $\mathrm{N}$-bridged drugs like triazolam (sedative agent) [1,5], estazolam and alprazolam (anxiolytic agents) $[1,6,7]$. The 1,3,4-thiadiazoles have attracted more interest to the researchers due to their prominent biological properties including anticonvulsant, anti-tubercular, anti-inflammatory, antileishmanial, antioxidant [8], antimicrobial and antidepressant activities [8,9]. There are also many drugs containing the 1,3,4-thiadiazole moiety such as acetazolamide, megazol and methazolamide [10]. Interestingly, some current reports showed that the fusion of the biolabile 1,2,4-triazole with 1,3,4-thiadiazole rings affords fused bicyclic compounds with improved biological activities than their corresponding non-bridged precursors. These include some ibuprofen-based 1,2,4-triazolo[3,4-b]-1,3,4thiadiazoles with enhanced anti-inflammatory and analgesic properties than precursor ibuprofen [11-13], and some naproxen-based 1,2,4-triazolo[3,4-b]-1,3,4-thiadiazoles with enhanced analgesic, ulcerogenic, anti-inflammatory, and lipid peroxidation activities than starting naproxen [14]. Furthermore, $\mathrm{N}$-(heteroarylmethyl) aniline derivatives with varied potent activities such as antimicrobial [15,16], antifungal, anticancer $[17,18]$, herbicidal [19] and analgesic [20] were also reported. Prompted by these observations and in continuation of our research for new bioactive fused heterocycles [21,22], we herein describe the synthesis of a new series of 6-arylaminomethyl-3substituted-1,2,4-triazolo[3,4-b]-1,3,4-thiadiazoles as promising antioxidant agents.

\section{Materials and Methods}

\section{Chemistry}

The Innovative DTC-967A apparatus was used to determine the melting points of the newly synthesized compounds and the results are uncorrected. The IR-spectra were recorded on Shimadzu FT-IR Prestige- 21 spectrophotometer in $\mathrm{KBr}$ pellets and are expressed in $\mathrm{cm}^{-1}$. The mass spectra were recorded on a Shimadzu LC-MS-8030 mass spectrometer operating at $70 \mathrm{eV}$. The ${ }^{1} \mathrm{H}-\mathrm{NMR}$ spectra were recorded on a Bruker Avance II $400 \mathrm{MHz}$ instrument in DMSO-d solvent and TMS as an internal standard. All chemical shift values were reported as $\delta(\mathrm{ppm})$, downfield from TMS. The Systronics spectrophotometer 106 was used to record the absorbance of DPPH radical scavenging antioxidant assay. The $\mathrm{C}, \mathrm{H}, \mathrm{N}$ analysis was carried out on Vario EL III Elemental Analyzer. The completion of the reaction and the purity of the compounds were monitored by 
TLC using Merck silica gel $60 \mathrm{~F}_{256}$ coated aluminum with hexane: ethylacetate $(2: 8)$ as the mobile phase.

General Procedure for the Synthesis of 6-arylaminomethyl3-substituted-1,2,4-triazolo[3,4-b]-1,3,4-thiadiazoles (5a-s)

The equimolar amounts of 1,2,4-triazoles (2) (0.01 mol) and anilinoacetic acids (4) in phosphorous oxychloride $(20 \mathrm{ml})$ were heated under reflux for 16 hours. After cooling, the resulting reaction mass was poured into crushed ice with stirring. Finally, powdered sodium bicarbonate was added portion wise till the $\mathrm{pH}$ of the reaction mixture was raised to 8 . The solid separated was filtered, washed thoroughly with cold water, dried and recrystallized from ethanol to give pure compounds $5 \mathrm{a}$-s. The spectral data of some selected illustrative compounds prepared according to this procedure are given below:

6-(p-Chloroanilinomethyl)-[1,2,4]triazolo $[3,4-b][1,3,4]$ thiadiazole (5a)

IR ( $\mathrm{KBr}, \mathrm{cm}^{-1}$ ): 3316 (NH stretch), 2968 (C-H stretch), 1642 $(\mathrm{C}=\mathrm{N}) .{ }^{1} \mathrm{H}-\mathrm{NMR}\left(400 \mathrm{MHz}, \mathrm{DMSO}-\mathrm{d}_{6}\right) *: \delta(\mathrm{ppm}) 4.64\left(\mathrm{~s}, 2 \mathrm{H}, \mathrm{CH}_{2}-\mathrm{N}\right)$, 6.67 (d, J=8.56 Hz, 2H, Ar-H), 7.11 (d, J=8.48 Hz, 2H, Ar-H), 9.36 (s, $1 \mathrm{H},=\mathrm{CH}-\mathrm{N}$ of triazolothiadiazole ring). LC-MS (m/z): $263.75\left(\mathrm{M}^{+}-\right.$ 1), $265.90\left(M^{+}+1\right)$

6-(Anilinomethyl)-3-methyl-[1,2,4]triazolo $[3,4-b][1,3,4]$ thiadiazole (5d)

IR ( $\mathrm{KBr}, \mathrm{cm}^{-1}$ ): 3358 (NH stretch), 2968 (C-H stretch), 1632 $(\mathrm{C}=\mathrm{N}) .{ }^{1} \mathrm{H}-\mathrm{NMR}\left(400 \mathrm{MHz}, \mathrm{DMSO}-\mathrm{d}_{6}\right): \delta(\mathrm{ppm}) 2.64\left(\mathrm{~s}, 3 \mathrm{H}, \mathrm{CH}_{3}\right)$, $4.62\left(\mathrm{~s}, 2 \mathrm{H}, \mathrm{CH}_{2}-\mathrm{N}\right), 6.65(\mathrm{~m}, 3 \mathrm{H}, \mathrm{Ar}-\mathrm{H}), 7.11(\mathrm{~m}, 2 \mathrm{H}, \mathrm{Ar}-\mathrm{H}), 7.47$ (s, broad, 1H, -NH). LC-MS (m/z): $246.00\left(\mathrm{M}^{+}+1\right)$.

6-(p-Chloroanilinomethyl)-3-methyl-[1,2,4]triazolo[3,4-b] $[1,3,4]$ thiadiazole $(5 e)$

IR (KBr, cm${ }^{-1}$ ): 3312 (NH stretch), 2974 (C-H stretch), 1624 $(\mathrm{C}=\mathrm{N}) .{ }^{1} \mathrm{H}-\mathrm{NMR}\left(400 \mathrm{MHz}, \mathrm{DMSO}-\mathrm{d}_{6}\right): \delta(\mathrm{ppm}) 2.64\left(\mathrm{~s}, 3 \mathrm{H}, \mathrm{CH}_{3}\right)$, 4.63 (s, 2H, -CH2-N), 6.67 (d, J=8.84 Hz, 2H, Ar-H), 6.88 (s, broad, $\left.{ }^{1} \mathrm{H},-\mathrm{NH}\right), 7.09$ (d, J=8.88 Hz, 2H, Ar-H). LC-MS (m/z): $279.90\left(\mathrm{M}^{+}+\right.$ 1), $281.90\left(\mathrm{M}^{+}+3\right)$.

6-(p-Fluoroanilinomethyl)-3-methyl-[1,2,4]triazolo[3,4-b] $[1,3,4]$ thiadiazole $(5 f)$

IR ( $\mathrm{KBr}, \mathrm{cm}^{-1}$ ): 3315 (NH stretch), 2922 (C-H stretch), 1614 $(\mathrm{C}=\mathrm{N}) .{ }^{1} \mathrm{H}-\mathrm{NMR}(400 \mathrm{MHz}, \mathrm{DMSO}-\mathrm{d} 6): \delta(\mathrm{ppm}) 2.66$ (s, 3H, $\mathrm{CH}_{3}$ ), 4.59 (s, 2H, $-\mathrm{CH}_{2}-\mathrm{N}$ ), 6.40-6.55 (broad, $\left.1 \mathrm{H}, \mathrm{NH}\right), 6.65(\mathrm{~m}, 2 \mathrm{H}, \mathrm{Ar}-\mathrm{H})$, $6.88(\mathrm{t}, 2 \mathrm{H}, \mathrm{Ar}-\mathrm{H})$. LC-MS (m/z): $264.02\left(\mathrm{M}^{+}+1\right)$.

6-(p-Methylanilinomethyl)-3-methyl-[1,2,4] triazolo[3,4-b] $[1,3,4]$ thiadiazole $(5 g)$

IR (KBr, cm${ }^{-1}$ ): 3406 (NH stretch), 2984 (C-H stretch), 1633 $(\mathrm{C}=\mathrm{N}) .{ }^{1} \mathrm{H}-\mathrm{NMR}\left(400 \mathrm{MHz}, \mathrm{DMSO}-\mathrm{d}_{6}\right) *: \delta(\mathrm{ppm}) 2.18\left(\mathrm{~s}, 3 \mathrm{H}, \mathrm{CH}_{3}\right)$, $2.67\left(\mathrm{~s}, 3 \mathrm{H},-\mathrm{CH}_{3}\right), 4.60\left(\mathrm{~s}, 2 \mathrm{H},-\mathrm{CH}_{2}-\mathrm{N}\right), 6.58(\mathrm{~d}, \mathrm{~J}=8.44 \mathrm{~Hz}, 2 \mathrm{H}, \mathrm{Ar}-\mathrm{H})$, $6.93(\mathrm{~d}, \mathrm{~J}=8.2 \mathrm{~Hz}, 2 \mathrm{H}, \mathrm{Ar}-\mathrm{H})$. LC-MS (m/z): $260.00\left(\mathrm{M}^{+}+1\right)$.
6-(Anilinomethyl)-3-ethyl-[1,2,4]triazolo[3,4-b][1,3,4] thiadiazole (5j)

IR ( $\mathrm{KBr}, \mathrm{cm}^{-1}$ ): 3346 (NH stretch), 2971 (C-H stretch), 1618 $(\mathrm{C}=\mathrm{N}) .{ }^{1} \mathrm{H}-\mathrm{NMR}\left(400 \mathrm{MHz}, \mathrm{DMSO}-\mathrm{d}_{6}\right) *: \delta(\mathrm{ppm}) 1.38\left(\mathrm{t}, 3 \mathrm{H}, \mathrm{CH}_{3}\right)$, $3.03\left(\mathrm{q}, 2 \mathrm{H},-\mathrm{CH}_{2}\right.$ ), $4.62(\mathrm{~s}, 2 \mathrm{H},-\mathrm{CH} 2-\mathrm{N}), 6.66(\mathrm{~m}, 3 \mathrm{H}, \mathrm{Ar}-\mathrm{H}), 7.11(\mathrm{~m}$, 2H, Ar-H). LC-MS (m/z): $260.01\left(\mathrm{M}^{+}+1\right)$.

6-(p-Fluoroanilinomethyl)-3-ethyl-[1,2,4]triazolo[3,4-b] $[1,3,4]$ thiadiazole $(51)$

IR ( $\mathrm{KBr}, \mathrm{cm}^{-1}$ ): 3319 (NH stretch), 2931 (C-H stretch), 1614 $(\mathrm{C}=\mathrm{N}) .{ }^{1} \mathrm{H}-\mathrm{NMR}(400 \mathrm{MHz}, \mathrm{DMSO}-\mathrm{d} 6) *$ : $\delta(\mathrm{ppm}) 1.40\left(\mathrm{t}, 3 \mathrm{H}, \mathrm{CH}_{3}\right)$, $3.03\left(\mathrm{q}, 2 \mathrm{H},-\mathrm{CH}_{2}\right), 4.61\left(\mathrm{~s}, 2 \mathrm{H},-\mathrm{CH}_{2}-\mathrm{N}\right), 6.66(\mathrm{~m}, 2 \mathrm{H}, \mathrm{Ar}-\mathrm{H}), 6.89(\mathrm{t}$, 2H, Ar-H). LC-MS (m/z): $278.05\left(\mathrm{M}^{+}+1\right)$.

6-(p-Methylanilinomethyl)-3-ethyl-[1,2,4]triazolo[3,4-b] $[1,3,4]$ thiadiazole $(5 \mathrm{~m})$

IR ( $\mathrm{KBr}, \mathrm{cm}^{-1}$ ): 3344 (NH stretch), 2978 (C-H stretch), 1614 $(\mathrm{C}=\mathrm{N}) .{ }^{1} \mathrm{H}-\mathrm{NMR}\left(400 \mathrm{MHz}, \mathrm{DMSO}-\mathrm{d}_{6}\right): \delta(\mathrm{ppm}) 1.39\left(\mathrm{t}, 3 \mathrm{H}, \mathrm{CH}_{3}\right), 2.17$ (s, $3 \mathrm{H},-\mathrm{CH}_{3}$ ), 3.04 (q, 2H, $-\mathrm{CH}_{2}$ ), 4.58 (s, 2H, $\left.-\mathrm{CH} 2-\mathrm{N}\right), 6.45$ (s, broad, $\left.{ }^{1} \mathrm{H},-\mathrm{NH}\right), 6.57$ (d, J=8.2 Hz, 2H, Ar-H), 6.92 (d, J=8.04 Hz, 2H, Ar-H). LC-MS (m/z): $274.05\left(\mathrm{M}^{+}+1\right)$.

6-(p-Chloroanilinomethyl)-3-propyl-[1,2,4]triazolo[3,4-b] $[1,3,4]$ thiadiazole $(5 p)$

IR (KBr, cm-1): 3304 (NH stretch), 2956 (C-H stretch), 1612 $(\mathrm{C}=\mathrm{N}) .{ }^{1} \mathrm{H}-\mathrm{NMR}\left(400 \mathrm{MHz}, \mathrm{DMSO}-\mathrm{d}_{6}\right): \delta(\mathrm{ppm}) 0.94\left(\mathrm{t}, 3 \mathrm{H}, \mathrm{CH}_{3}\right), 1.81$ ( $\mathrm{m}, 2 \mathrm{H},-\mathrm{CH} 2-), 2.98\left(\mathrm{t}, 2 \mathrm{H},-\mathrm{CH}_{2}-\right), 4.61\left(\mathrm{~s}, 2 \mathrm{H},-\mathrm{CH}_{2}-\mathrm{N}\right), 6.64(\mathrm{~d}$, $\mathrm{J}=8.84 \mathrm{~Hz}, 2 \mathrm{H}, \mathrm{Ar}-\mathrm{H}$ ), 7.06 (d, J=6.96 Hz, 2H, Ar-H), 7.46 (s, broad, 1H, -NH). LC-MS (m/z): $309.9\left(\mathrm{M}^{+}+1\right), 311.9\left(\mathrm{M}^{+}+3\right)$.

6-(p-methylanilinomethyl)-3-propyl-[1,2,4]triazolo[3,4-b] $[1,3,4]$ thiadiazole $(5 r)$

IR (KBr, cm-1): 3311 (NH stretch), 2929 (C-H stretch), 1614 $(\mathrm{C}=\mathrm{N}) .{ }^{1} \mathrm{H}-\mathrm{NMR}\left(400 \mathrm{MHz}, \mathrm{DMSO}-\mathrm{d}_{6}\right)^{*}: \delta(\mathrm{ppm}) 1.00\left(\mathrm{t}, 3 \mathrm{H}, \mathrm{CH}_{3}\right)$, $1.84\left(\mathrm{~m}, 2 \mathrm{H},-\mathrm{CH}_{2}\right), 2.17\left(\mathrm{~s}, 3 \mathrm{H},-\mathrm{CH}_{3}\right), 2.99(\mathrm{t}, 2 \mathrm{H},-\mathrm{CH} 2), 4.58(\mathrm{~s}, 2 \mathrm{H}$, -CH2-N), 6.58 (d, J=8.4 Hz, 2H, Ar-H), 6.92 (d, J=8.2 Hz, 2H, Ar-H). LC-MS (m/z): $288.05\left(\mathrm{M}^{+}+1\right)$.

*NH peak was not seen probably due to the rapid exchange between this $\mathrm{NH}$ with D2O that might be present as impurities in the DMSO-d6 solvent used to record these spectra.

\section{Antioxidant activity}

The DPPH radical scavenging assay was carried out by the modified method of Mensor et al. [23]. The working test sample solution $(1 \mathrm{mg} / \mathrm{ml})$ was made in DMSO. $150 \mu$ l was taken from each test sample solution in a test tube, diluted with ethanol up to $2.5 \mathrm{ml}$, then freshly prepared DPPH solution ( $3 \mathrm{mM}$ in ethanol) (1ml) was added to each test tube and shaken well. After 30 min of incubation in dark condition at the ambient room temperature, the absorbance of the sample was read at $518 \mathrm{~nm}$ against the absorbance of the control (Ac) prepared in the same way as the sample solution, 
except that $150 \mu$ l of test sample was replaced by ethanol solvent. The DPPH radical scavenging activity was calculated as follows: $\%$ radical scavenged $=[(\mathrm{Ac}-\mathrm{As}) / \mathrm{Ac}] \mathrm{x}$ 100. The experiments were replicated thrice and the results were presented as their mean value \pm SD.

\section{Result and Discussion}

\section{Chemistry}

The synthesis of title compounds (5) is as outlined in the Figure 1. The key starting material thiocarbohydrazide (1) was prepared by reacting hydrazine hydrate with carbon disulfide [24]. The cyclocondensation of thiocarbohydrazide (1) with different carboxylic acids (formic, acetic, propionic and butyric acids) under reflux condition afforded 5-substituted-4-amino-3-mercapto-1,2,4- triazoles (2) [25,26]. The required substituted anilinoacetic acids (4) were synthesized by hydrolyzing their corresponding esters (3) in aqueous $\mathrm{NaOH}$ [27]. The latter compounds (3) were prepared by refluxing various anilines, sodium acetate and ethyl chloroacetate in ethanol solvent [28]. The cyclocondensation of 5-substituted-4amino-3-mercapto-1,2,4-triazoles (2) with anilinoacetic acids (4) using phosphorous oxychloride as a condensing agent afforded new bicyclic compounds 1,2,4-triazolo[3,4-b]-1,3,4-thiadiazoles carrying $\mathrm{N}$-methyl aniline moiety (5). The ${ }^{1} \mathrm{H}-\mathrm{NMR}$, IR, Mass spectral and elemental analysis confirmed the proposed structure of the newly synthesized compounds. The characterization data of the title compounds (5) is given in Table 1. The detailed description of the spectra data of compound $5 \mathrm{~h}$ taken as a typical example is given below.

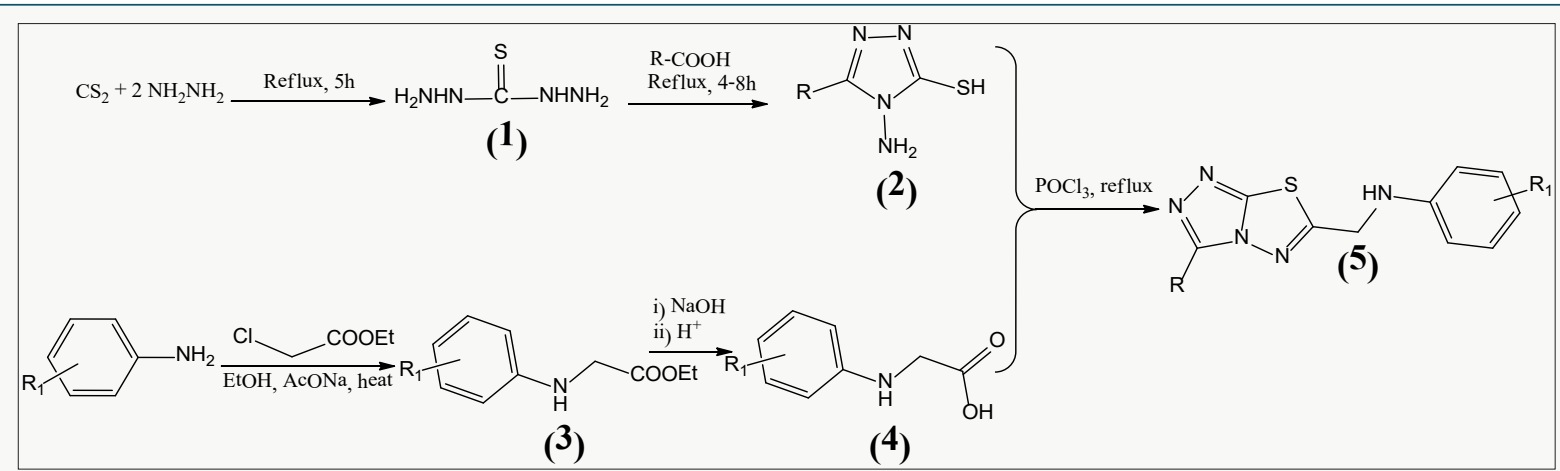

Figure 1: Outline for the synthesis of novel 1,2,4-triazolo[3,4-b]-1,3,4-thiadiazoles (5a-s).

$\mathrm{R}=\mathrm{H}, \mathrm{CH}_{3}, \mathrm{C}_{2} \mathrm{H} 5, \mathrm{C}_{3} \mathrm{H}_{7} ; \mathrm{R} 1=\mathrm{H}, 4-\mathrm{Cl}, 4-\mathrm{F}, 4-\mathrm{CH}_{3}, 4-\mathrm{OCH}_{3}, 3,4-\mathrm{Cl}_{2}$

Table 1: Characterization data of 6-arylaminomethyl-3-substituted-1,2,4-triazolo[3,4-b]-1,3,4-thiadiazoles (5a-s).

\begin{tabular}{|c|c|c|c|c|c|c|c|}
\hline \multirow{2}{*}{ Compound } & \multirow{2}{*}{$\mathbf{R}$} & \multirow{2}{*}{$\mathbf{R} 1$} & \multirow{2}{*}{ M.P (oC) (Yield \%) } & \multirow{2}{*}{$\begin{array}{l}\text { Molecular Formula (Mol. } \\
\text { Wt) }\end{array}$} & \multicolumn{3}{|c|}{ Elemental Analysis Found (Calculated) (\%) } \\
\hline & & & & & C & $\mathbf{H}$ & $\mathbf{N}$ \\
\hline $5 a$ & $\mathrm{H}$ & $4-\mathrm{Cl}$ & $102-104(48)$ & $\mathrm{C}_{10} \mathrm{H}_{8} \mathrm{ClN}{ }_{5} \mathrm{~S}(265.72)$ & $45.18(45.20)$ & $3.08(3.03)$ & $26.24(26.36)$ \\
\hline $5 \mathrm{~b}$ & $\mathrm{H}$ & $4-\mathrm{F}$ & $108-110(40)$ & $\mathrm{C}_{10} \mathrm{H}_{8} \mathrm{FN} 5 \mathrm{~S}(249.27)$ & $48.16(48.18)$ & $3.37(3.23)$ & $28.08(28.10)$ \\
\hline $5 c$ & $\mathrm{H}$ & $4-\mathrm{CH}_{3}$ & 100-102 (32) & $\mathrm{C}_{11} \mathrm{H}_{11} \mathrm{~N}_{5} \mathrm{~S}(245.30)$ & $53.91(53.86)$ & $4.50(4.52)$ & $28.62(28.55)$ \\
\hline $5 \mathrm{~d}$ & $\mathrm{CH}_{3}$ & $\mathrm{H}$ & $158-160(70)$ & $\mathrm{C}_{11} \mathrm{H}_{11} \mathrm{~N}_{5} \mathrm{~S}(245.30)$ & $53.88(53.86)$ & $4.48(4.52)$ & $28.64(28.55)$ \\
\hline $5 e$ & $\mathrm{CH}_{3}$ & 4-Cl & $102-104(45)$ & $\mathrm{C}_{11} \mathrm{H}_{10} \mathrm{ClN}_{5} \mathrm{~S}(279.75)$ & $47.11(47.23)$ & $3.62(3.60)$ & $25.15(25.03)$ \\
\hline $5 f$ & $\mathrm{CH}_{3}$ & $4-\mathrm{F}$ & $108-110(80)$ & $\mathrm{C}_{11} \mathrm{H}_{10} \mathrm{FN}_{5} \mathrm{~S}(263.29)$ & $50.23(50.18)$ & $3.90(3.83)$ & $26.52(26.60)$ \\
\hline $5 \mathrm{~g}$ & $\mathrm{CH}_{3}$ & $4-\mathrm{CH}_{3}$ & $100-102(91)$ & $\mathrm{C}_{12} \mathrm{H}_{13} \mathrm{~N}_{5} \mathrm{~S}(259.33)$ & $55.47(55.58)$ & $5.09(5.05)$ & $26.94(27.01)$ \\
\hline $5 \mathrm{~h}$ & $\mathrm{CH}_{3}$ & $4-\mathrm{OCH}_{3}$ & 80-82 (82) & $\mathrm{C}_{12} \mathrm{H}_{13} \mathrm{~N}_{5} \mathrm{OS}(275.33)$ & $52.26(52.35)$ & $4.87(4.76)$ & $25.48(25.44)$ \\
\hline $5 \mathrm{i}$ & $\mathrm{CH}_{3}$ & $3,4-\mathrm{Cl}_{2}$ & 101-103 (79) & $\mathrm{C}_{11} \mathrm{H}_{9} \mathrm{C}_{12} \mathrm{~N}_{5} \mathrm{~S}(314.19)$ & $42.16(42.05)$ & $3.04(2.89)$ & $22.12(22.29)$ \\
\hline $5 j$ & $\mathrm{C}_{2} \mathrm{H}_{5}$ & $\mathrm{H}$ & $98-100(66)$ & $\mathrm{C}_{12} \mathrm{H}_{13} \mathrm{~N}_{5} \mathrm{~S}(259.33)$ & $55.62(55.58)$ & $5.18(5.05)$ & $26.95(27.01)$ \\
\hline $5 \mathrm{k}$ & $\mathrm{C}_{2} \mathrm{H}_{5}$ & 4-Cl & $79-81(71)$ & $\mathrm{C}_{12} \mathrm{H}_{12} \mathrm{ClN}_{5} \mathrm{~S}(293.78)$ & $49.18(49.06)$ & $4.19(4.12)$ & $23.78(23.84)$ \\
\hline 51 & $\mathrm{C}_{2} \mathrm{H}_{5}$ & $4-\mathrm{F}$ & $118-120(69)$ & $\mathrm{C}_{12} \mathrm{H}_{12} \mathrm{FN}_{5} \mathrm{~S}(277.32)$ & $51.82(51.97)$ & $4.42(4.36)$ & $25.19(25.25)$ \\
\hline $5 \mathrm{~m}$ & $\mathrm{C}_{2} \mathrm{H}_{5}$ & $4-\mathrm{CH}_{3}$ & 80-82 (82) & $\mathrm{C}_{13} \mathrm{H}_{15} \mathrm{~N}_{5} \mathrm{~S}(273.36)$ & $57.19(57.12)$ & $5.48(5.53)$ & $25.69(25.62)$ \\
\hline $5 n$ & $\mathrm{C}_{3} \mathrm{H}_{7}$ & $\mathrm{H}$ & $76-78(48)$ & $\mathrm{C}_{13} \mathrm{H}_{15} \mathrm{~N}_{5} \mathrm{~S}(273.36)$ & $57.03(57.12)$ & $5.44(5.53)$ & $25.75(25.62)$ \\
\hline 50 & $\mathrm{C}_{3} \mathrm{H}_{7}$ & $4-\mathrm{Br}$ & $82-84(78)$ & $\mathrm{C}_{13} \mathrm{H}_{14} \mathrm{BrN}_{5} \mathrm{~S}(352.25)$ & $44.47(44.33)$ & $4.08(4.01)$ & $19.76(19.88)$ \\
\hline $5 p$ & $\mathrm{C}_{3} \mathrm{H}_{7}$ & 4-Cl & $80-82(92)$ & $\mathrm{C}_{13} \mathrm{H}_{14} \mathrm{ClN}_{5} \mathrm{~S}(307.80)$ & $50.61(50.73)$ & $4.49(4.58)$ & $22.84(22.75)$ \\
\hline $5 q$ & $\mathrm{C}_{3} \mathrm{H}_{7}$ & $4-\mathrm{F}$ & $81-83(77)$ & $\mathrm{C}_{13} \mathrm{H}_{14} \mathrm{FN}_{5} \mathrm{~S}(291.35)$ & $53.52(53.59)$ & $4.76(4.84)$ & $24.12(24.04)$ \\
\hline $5 r$ & $\mathrm{C}_{3} \mathrm{H}_{7}$ & $4-\mathrm{CH}_{3}$ & 100-102 (97) & $\mathrm{C}_{14} \mathrm{H}_{17} \mathrm{~N}_{5} \mathrm{~S}(287.38)$ & $58.64(58.51)$ & $5.81(5.96)$ & $24.43(24.37)$ \\
\hline $5 \mathrm{~s}$ & $\mathrm{C}_{3} \mathrm{H}_{7}$ & $4-\mathrm{OCH}_{3}$ & $98-100(76)$ & $\mathrm{C}_{14} \mathrm{H}_{17} \mathrm{~N}_{5} \mathrm{OS}(303.38)$ & $55.38(55.42)$ & $5.74(5.65)$ & $23.12(23.08)$ \\
\hline
\end{tabular}


In the IR spectrum of 6-(p-methoxyanilinomethyl)-3methyl-[1,2,4] triazolo[3,4-b][1,3,4]thiadiazole (5h) the absorption band corresponding to $\mathrm{NH}$ stretching was observed at $3317 \mathrm{~cm}^{-1}$, $\mathrm{C}-\mathrm{H}$ stretching bands were seen at $3047 \mathrm{~cm}^{-1}$ and $2924 \mathrm{~cm}^{-1}$ while the $\mathrm{C}=\mathrm{N}$ stretching band appeared at $1614 \mathrm{~cm}^{-1}$. The formation of fused bicyclic compounds (5) was further confirmed by the absence of - $\mathrm{OH}$, $-\mathrm{SH}$ and - $\mathrm{NH} 2$ peaks of the precursors anilino acetic acids and 4-amino-3-mercapto-1,2,4-triazoles in the condensed products, thereby confirming their involvement in the formation of bicyclic triazolothiadiazole derivatives (5). Furthermore, the appearance of $-\mathrm{CH}_{2}-\mathrm{NH}$ - peaks in the proton NMR spectra of title compounds (5) was an additional proof for the formation of N-bridged bicyclic compounds (5). In the ${ }^{1} \mathrm{H}$-NMR spectrum of $\quad 6$-(p-methoxyanilinomethyl)-3-methyl-[1,2,4]triazolo[3,4-b] $[1,3,4]$ thiadiazole $(5 \mathrm{~h})$ (Figure 2a,2b), methyl protons $\left(-\mathrm{CH}_{3}\right)$ appeared as a singlet at $\delta 2.65 \mathrm{ppm}$ integrating for three protons, methoxy protons $\left(-\mathrm{OCH}_{3}\right)$ came into resonance as a singlet at $\delta 3.67$ ppm integrating for three protons, methylene $\left(-\mathrm{CH}_{2}-\mathrm{N}\right)$ protons resonated as a singlet at $\delta 4.55 \mathrm{ppm}$ integrating for two protons. The peak at $\delta 6.15 \mathrm{ppm}$ (broad band) integrating for one proton was assigned to $\mathrm{NH}$ peak. Two doublets centered at $\delta 6.62 \mathrm{ppm}(\mathrm{J}=8.84$ $\mathrm{Hz})$ and at $\delta 6.72 \mathrm{ppm}(\mathrm{J}=8.76 \mathrm{~Hz})$ integrating for two protons each were attributed to ortho and meta protons of p-methoxyphenyl ring respectively. The mass spectrum of this compound $5 \mathrm{~h}$ (Figure 3) showed molecular ion peak at $\mathrm{m} / \mathrm{z} 275.80\left(\mathrm{M}^{+}+1\right)$, which is in conformity with its molecular formula $\mathrm{C}_{12} \mathrm{H}_{13} \mathrm{~N}_{5} \mathrm{OS}$.

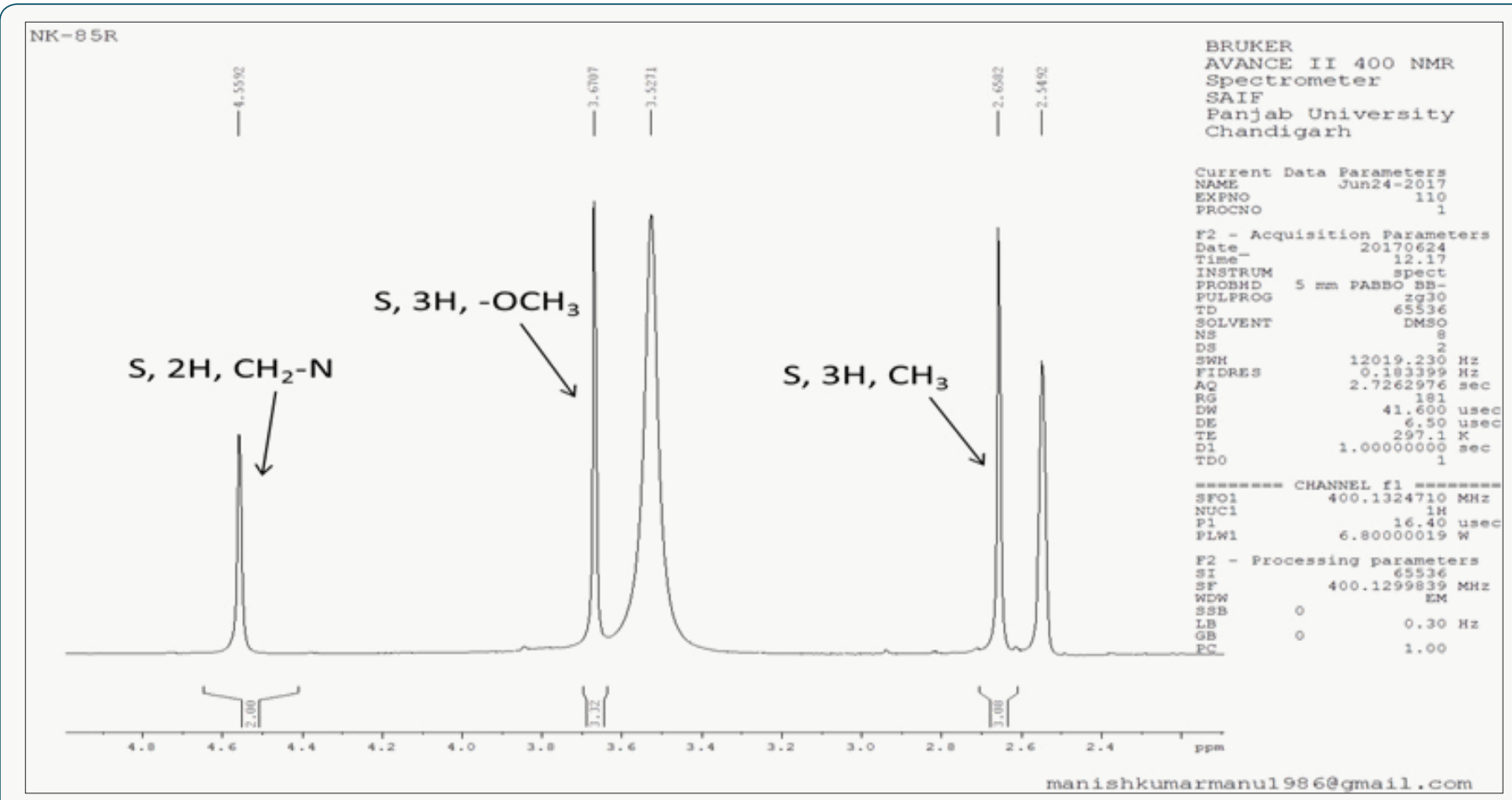

Figure 2b: Aliphatic region of 1H NMR of 6-(p-methoxyanilinomethyl)-3-methyl-[1,2,4]triazolo[3,4-b][1,3,4]thiadiazole (5h).

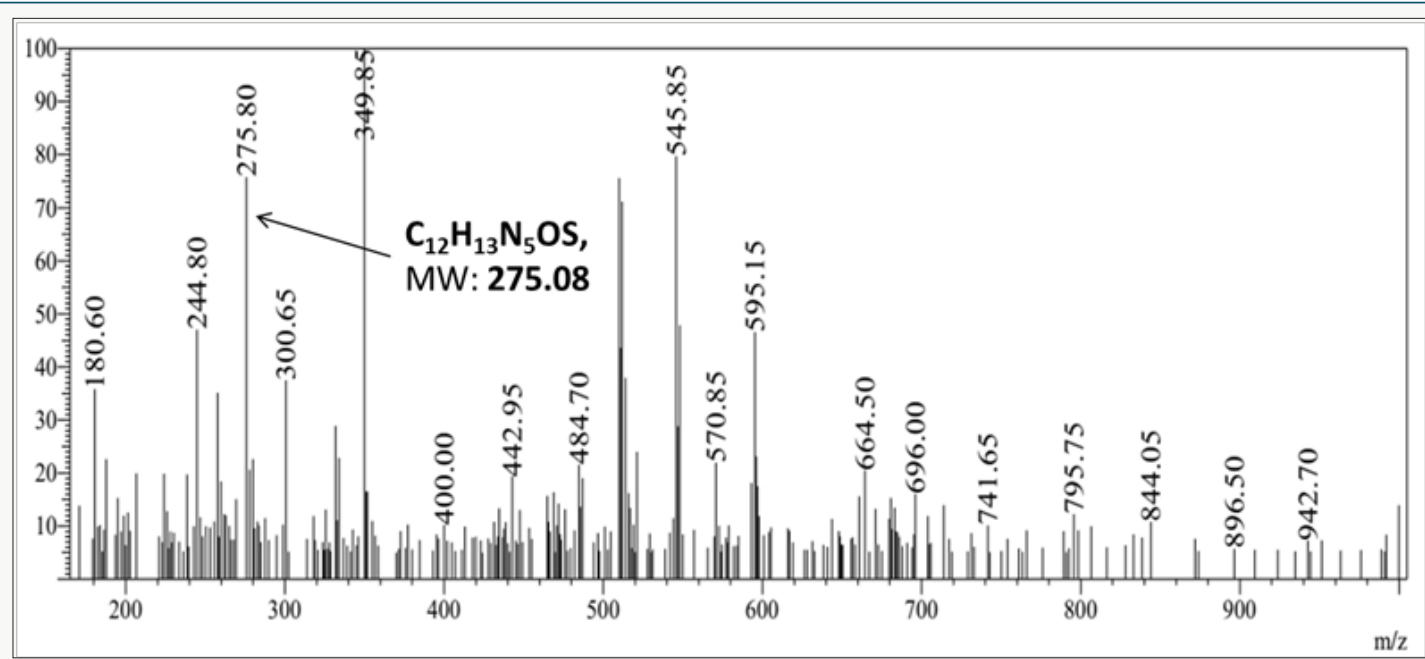

Figure 3: Mass spectrum of 6-(p-methoxyanilinomethyl)-3-methyl-[1,2,4]triazolo[3,4-b][1,3,4]thiadiazole (5h). 


\section{Antioxidant activity}

The DPPH free radical scavenging assay was used to screen for the antioxidant activity of the newly synthesized compounds 5a-s. The extent of discoloration of DPPH stable radical from violet to colorless or pale yellow indicated the scavenging potential of the tested compounds. The results as presented in Table 2 showed that methoxy substituted compounds on phenyl ring $\left(\mathrm{R}_{1}=4-\mathrm{OCH} 3\right)$ (5s, 5h), and unsubstituted compounds on 1,2,4-triazole moiety of triazolo-thiadiazole ring $(\mathrm{R}=\mathrm{H})(5 \mathrm{a}, 5 \mathrm{~b}, 5 \mathrm{c})$ exhibited very good antioxidant activities in the series with respectively $73.7 \%$, $67.9 \%$ and $70.7 \%, 67.8 \%, 71.4 \%$ DPPH radical scavenger activity comparable to that of standard butylated hydroxyanisole (BHA) (87.1\%).

Table 2: DPPH scavenging activity of compounds 5a-s.

\begin{tabular}{|c|c|}
\hline Compound & Percentage DPPH Inhibition (\%)a \\
\hline $5 \mathrm{a}$ & $70.7 \pm 0.20$ \\
\hline $5 \mathrm{~b}$ & $67.8 \pm 0.30$ \\
\hline $5 \mathrm{c}$ & $71.4 \pm 0.17$ \\
\hline $5 \mathrm{~d}$ & $53.5 \pm 0.37$ \\
\hline $5 \mathrm{e}$ & $25.5 \pm 0.10$ \\
\hline $5 \mathrm{f}$ & $39.6 \pm 0.26$ \\
\hline $5 \mathrm{~g}$ & $37.5 \pm 0.17$ \\
\hline $5 \mathrm{~h}$ & $67.9 \pm 0.20$ \\
\hline $5 \mathrm{i}$ & $23.4 \pm 0.10$ \\
\hline $5 \mathrm{j}$ & $44.5 \pm 0.17$ \\
\hline $5 \mathrm{k}$ & $29.6 \pm 0.10$ \\
\hline $5 \mathrm{l}$ & $36.7 \pm 0.20$ \\
\hline $5 \mathrm{~m}$ & $52.6 \pm 0.17$ \\
\hline $5 \mathrm{n}$ & $49.5 \pm 0.10$ \\
\hline $5 \mathrm{o}$ & $40.1 \pm 0.17$ \\
\hline $5 \mathrm{p}$ & $40.3 \pm 0.17$ \\
\hline $5 \mathrm{q}$ & $37.3 \pm 0.10$ \\
\hline $5 \mathrm{r}$ & $43.3 \pm 0.26$ \\
\hline $5 \mathrm{~s}$ & $73.7 \pm 0.26$ \\
\hline $8 H A$ & $87.1 \pm 0.36$ \\
\hline & \\
\hline
\end{tabular}

\section{Conclusion}

In the present study, we achieved the synthesis of a new series of 1,2,4-triazolo[3,4-b]-1,3,4-thiadiazoles bearing N-methylaniline moiety. These newly synthesized compounds were characterized by analytical and spectroscopic methods. They were also screened for their antioxidant activity by DPPH free radical scavenging assay. Compounds $5 \mathrm{a}, 5 \mathrm{c}$ and $5 \mathrm{~s}$ were found to be most potent antioxidant agents in the series.

\section{Acknowledgement}

Authors are thankful to the Head, USIC-Mangalore University and SAIF-Punjab University for providing facilities for Spectral data.
One of the authors Jean Baptiste Nkurunziza is thankful to Indian Council for Culture Relations (ICCR) for providing a Scholarship.

\section{References}

1. Arshad M (2014) An insight to the synthetically obtained triazole possessing numerous biological activities. Int J Pharm Pharm Sci 6(9): 16-23.

2. Howell A, Robertson JFR, Vergote I (2003) A review of the efficacy of anastrozole in postmenopausal women with advanced breast cancer with visceral metastases. Breast Cancer Res Treat 82(3): 215-222.

3. Feld JJ, Jacobson IM, Sulkowski MS, Poordad F, Tatsch F, et al. (2017) Ribavirin revisited in the era of direct-acting antiviral therapy for hepatitis C virus infection. Liver Int 37(1): 5-18.

4. Jagdish KS, Ganguly S, Kaushik A (2014) Synthesis and antimicrobial activity of some novel fused heterocyclic 1,2,4-triazolo[3,4-b][1,3,4] thiadiazine derivatives. J Adv Pharm Technol Res 5(2): 90-95.

5. Kim D, Lee S, Pyeon T, Jeong S (2015) Use of triazolam and alprazolam as premedication for general anesthesia. Korean J Anesthesiol 68(4): 346351.

6. Godhani DR, Jogel AA, Sanghani AM, Mehta JP (2015) Synthesis and biological screening of 1,2,4-triazole derivatives. Indian J Chem 54B: 556-564.

7. Zhou CH, Wang Y (2012) Recent researches in triazole compounds as medicinal drugs. Curr Med Chem 19(2): 239-280.

8. Kushwaha N, Kushwaha SKS, Rai AK (2012) Biological activities of thiadiazole derivatives: A review. Int J Chem Tech Res 4(2): 517-531.

9. Siddiqui N, Ahuja P, Ahsan W, Pandeya SN, Alam MS (2009) Thiadiazoles: Progress report on biological activities. J Chem Pharm Res 1(1):19-30.

10. Jain AK, Sharma S, Vaidya A, Ravichandran V, Agrawal RK (2013) 1,3,4-Thiadiazole and its derivatives: A review on recent progress in biological activities. Chem Biol Drug Des 81(5): 557-576.

11. Amir M, Kumar H, Javed SA (2007) Synthesis and pharmacological evaluation of condensed heterocyclic 6-substituted-1,2,4-triazolo[3,4b]-1,3,4-thiadiazole derivatives of naproxen. Bioorg Med Chem Lett 17(16): 4504-4508.

12. Sujith KV, Kalluraya B, Adhikari A, Ravikumar J (2011) Microwave mediated synthesis of non-carboxylic analogues of ibuprofen with improved pharmacological activity. Chin Chem Lett 22(5): 508-510.

13. Sujith KV, Kalluraya B, Adhikari A, Vijayanarayana K (2012) Microwavemediated synthesis of triazolothiadiazoles as anti-inflammatory, analgesic, and anti-oxidant agents. Med Chem Res 21(5): 543-551.

14. Amir A, Kumar H, Javed SA (2008) Condensed bridgehead nitrogen heterocyclic system: Synthesis and pharmacological activities of 1,2,4-triazolo-[3,4-b]-1,3,4-thiadiazole derivatives of ibuprofen and biphenyl-4-yloxy acetic acid. Eur J Med Chem 43(10): 2056-2066.

15. Prasanna Kumar BN, Mohana KN, Mallesha L, Rekha ND (2013) Synthesis of (E)-2-(arylbenzylidene)-2-((4-methoxyphenyl)amino) acetohydrazide derivatives and their antimicrobial activity. Current Chemistry Letters 2: 167-176.

16. Karthikeyan MS, Holla BS, Kalluraya B, Kumari NS (2007) Biological studies of some 2,4-dichloro-5-fluorophenyl containing triazolothiadiazoles. Monatshefte fur Chemie 138(12): 1309-1316.

17. Kouznetsov VV, Mendez LYV, Sortino M, Vasquez Y, Gupta MP, et al. (2008) Antifungal and cytotoxic activities of some N-substituted aniline derivatives bearing a hetaryl fragment. Bioorganic Med Chem 16(2): 794-809. 
18. Kouznetsov VV, Zacchino SA, Sortino M, Méndez LYV, Gupta MP (2012) Cytotoxic and antifungal activities of diverse $\alpha$-naphthylamine derivatives. Sci Pharm 80(4): 867-877.

19. Katke SA, Amrutkar SV, Bhor RJ, Khairnar MV (2011) Synthesis of biologically active 2-chloro-n-alkyl/aryl acetamide derivatives. Int J Pharm Sci Res 2(7): 148-156.

20. Mariappan G, Bhuyan NR, Kumar P, Kumar D, Murali K (2011) Synthesis and biological evaluation of Mannich bases of benzimidazole derivatives. Indian J Chem 50B: 1216-1219.

21. Ramaprasad GC, Kalluraya B, Kumar BS (2014) Microwave-assisted synthesis of triazolothiadiazole analogs as anticancer agents. Med Chem Res 23: 3644-3651.

22. Ramaprasad GC, Kalluraya B, Kumar BS, Mallya S (2012) Microwave assisted synthesis of triazolothiadiazole analogues as anticancer and antibacterial agents. Der Pharma Chemica 4(3): 1026-1032.

23. Mensor LI, Menezes FS, leitao GG, Reis AS, Dos Santos T, et al. (2001) Screening of Brazilian plant extracts for antioxidant activity by the use of DPPH Free Radical Method. Phytother Res 15(2): 127-130.
24. Abdullah JH, Yahya TAA, Al Ghorafi MAH, Yassin SH (2014) Synthesis and evaluation of new pyrazoline and thiazolidinone derivatives as anticancer activity. Der Pharma Chemica 6(6): 203-210.

25. McCarrick RM, Eltzroth MJ, Squattrito PJ (2000) Coordination geometries of bis(4-amino-3-alkyl-1,2,4-triazole-5-thione) complexes of first-row transition metals: crystal structures of complexes with propyl and hydrogen in the 3-position. Relationship to the 3-methyl and 3-ethyl analogs. Inorganica Chim Acta 311(1-2): 95-105.

26. Bazian A, Taheri M, Alavi H (2014) Synthesis of 4'-[3-methyl-5thioxo-1H-1,2,4-triazol-4(5 H) -yl]-2',5'-diphenyl-2',4'-dihydro spiro[indolin-3,3'[1,2,4]triazol]-2-one derivatives. Russ J Gen Chem 84(3): 586-582.

27. Patel YM, Patel KC (2015) Synthesis and biological evaluation of new sydnone based derivatives. J Saudi Chem Soc 19(2): 193-199.

28. Fang Y, Wu C, Larock RC, Shi F (2011) Synthesis of $2 \mathrm{H}$-indazoles by the [3 $+2]$ dipolar cycloaddition of sydnones with arynes. J Org Chem 76(21): 8840-8851.

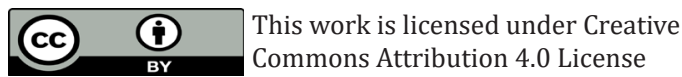

To Submit Your Article Click Here:

Submit Article

DOI: $10.32474 /$ AOICS.2018.02.000135

\section{AOICS}

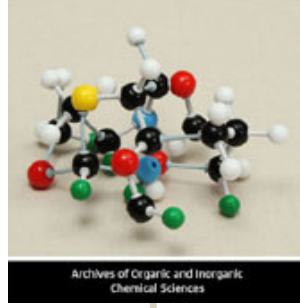

Archives of Organic and Inorganic Chemical Sciences

\section{Assets of Publishing with us}

- Global archiving of articles

- Immediate, unrestricted online access

- Rigorous Peer Review Process

- Authors Retain Copyrights

- Unique DOI for all articles 